\title{
CSF biochemical correlates of mixed affective states
}

\author{
R. Tandon', S. M. Channabasavanna ${ }^{2}$, \\ J. F. Greden' \\ 'University of Michigan Medical Center (Head: \\ Professor John F. Greden), Ann Arbor, Michigan, \\ USA, ${ }^{2}$ National Institute of Mental Health and \\ Neuro-Sciences, (NIMHANS), (Head: Professor \\ R. Srinivasa Moorthy), Bangalore, India
}

\begin{abstract}
To evaluate the question of whether "mixed" bipolar disorder is a distinct entity, we compared selected cerebrospinal fluid (CSF) biochemical parameters from patients with bipolar disorder, mixed, to those with mania and major depression. Fourteen patients in each category (DSM-III) were studied with regard to CSF HVA, SHIAA, sodium, potassium, calcium, and magnesium levels under carefully controlled conditions. CSF HVA, 5HIAA, and sodium were found to be significantly higher in manics than in major depressives. Discriminant analysis of the biochemical variables of the mixed affective group identified two biochemically distinct and clinically different subgroups of seven patients each, one resembling the manic group and the other the major depressive group. These findings suggest that mixed affective states do not exist as a separate entity, but are compsed of two subgroups obtained from the manic and major depressive categories.
\end{abstract}

Received August 29, 1987; accepted for publication February 27, 1988

The simultaneous existence of manic and depressive symptoms in affectively disordered patients was first described by Kraepelin (1), who called this condition a "mixed state" and included it as a subcategory of "manic-depressive insanity" (2). Kraepelin described six types of mixed affective states and emphasized that they often pose considerable diagnostic difficulties. Since that time, this diagnostic entity has generated much controversy.

According to the conventional "bipolar model" of affective disorders, depression and mania represent two extremes of an affective spectrum, with euthymia an intermediate condition (2-6). Viewed from this perspective, mixed affective states with an admixture of manic and depressive symptomatology should be impossible, as manic and depressive syndromes would be diametrically opposite conditions and mutually exclusive. Nev- ertheless, clinicians continue to encounter affective patients whose clinical profile is characterized by an admixture of manic and depressive symptoms. Perhaps because of such clinical "face validity", this diagnostic category has been included as a separate entity in all major international classification systems since the Kraepelin era. Both DSM-III (7) and ICD-9 (8) currently have four-digit denominations for this category. The existence of mixed affective states has prompted certain observers (9-12) to emphasize the viability of the "unipolar" or "continuum" model of manic-depressive illness, wherein depression represents mild illness and mania a more severe manifestation, with mixed states representing the intermediate "switch process".

While mixed affective states are of considerable clinical and theoretical importance, there have been few systematic studies of this entity. 
Most previous investigations in this area have been anecdotal observations or single case reports $(1,13,14)$, theoretical discussions $(4,11$, $14-16)$, studies of its incidence $(4,12)$, phenomenological descriptions $(10,17)$ and an evaluation of lithium resistance in this entity (18). The dearth of literature is particularly evident in the area of pathophysiological aspects, where the only systematic studies have been intensive biochemical investigations of the "switch process" in a few rapidly cycling bipolar patients $(10,19)$. These studies have revealed a functional increase of catecholamines prior to the switch from depression to mania. The present study was conducted in an effort to evaluate selected biochemical parameters in patients with mixed affective states and compare them with those with mania and major depression.

Alterations in the functional activity of brain neurotransmitter amines appear to be involved in the pathogenesis of affective disorders. Deficiencies of the monoamines (specifically norepinephrine, dopamine, and serotonin) at central synapses have been linked with depression, and excesses with mania $(6,20-23)$. Metabolites of these CNS neurotransmitters have been measured in the cerebrospinal fluid (CSF) as an indirect method of assessing central neurochemical function. The compounds most often measured are the predominant metabolites of norepinephrine, dopamine and serotonin (5-HT): 3-methoxy4-hydroxyphenylglycol (MHPG), homovanillic acid (HVA), and 5-hydroxyindoleacetic acid (5-HIAA), respectively. Over $30 \mathrm{CSF}$ studies in affective disorders have been conducted, and as recent comprehensive reviews $(24,25)$ have pointed out, the results have not been consistent. Nevertheless, CSF HVA levels have generally been found to be decreased (26-33) to normal (34-38) in depression, and normal $(39,40)$ to increased $(33,35,37,41)$ in mania. Similarly, 5-HIAA levels have been reported to be significantly decreased $(21,30,31,41,42)$ to normal $(27-29,32-36,38)$ in depression; and reduced $(41)$, normal $(35,42)$ or increased (33) in mania. Several studies of electrolyte (principally the cations sodium, potassium, calcium and magnesium) metabolism in affective disorders also have been conducted, with inconclusive results. While normal conditions appear to be the general rule (43), decreased CSF sodium levels in depression compared with mania were reported in one study (44).

The present study was conducted in an effort to elucidate the CSF biochemical profile (HVA, S-HIAA, sodium, potassium, calcium and magnesium) of mixed affective states and to compare it with those of mania and major depression. MHPG was not assayed because of laboratory technical difficulties with its assay at that time. The specific aim was to compare mixed affective states with mania and major depression and evaluate whether this condition constitutes a distinct biochemical entity different from mania and major depressive disorder. Another question that the study addressed was that of the possible clinical subcategorization of the mixed affective group and an investigation of the relationship between the clinical and biochemical categorization of mixed affective states. To our knowledge, this is the first systematic CSF study of mixed affective states.

\section{Material and methods}

\section{Subjects}

Subjects included 42 patients with mood disorders, 14 each in the categories of mania, major depression, and bipolar disorder, mixed. The manic group consisted of 10 male and 4 female patients aged $28.2 \pm 8.8$ years (mean $\pm \mathrm{SD}$ ); the major depressive group of 9 men and 5 women aged $35.6 \pm 9.8$ years; and the mixed affective group of 8 male and 6 female patients aged 29.4 \pm 8.9 years. There were no significant sex-distribution differences between the three groups $\left(\chi^{2}\right.$ $=0.62 ; \mathrm{df}=2$ ). Although the patients in the major depressive category were older than those in the other two categories, this difference was not statistically significant $(F=2.50, \mathrm{df}=$ 2,39 ). In the major depression group, the 8 unipolar and 6 bipolar subjects were not separated, as it has been suggested that there are no significant differences between them with regard to these CSF metabolites (33), and because of sample cell sizes.

A comprehensive psychiatric evaluation was 
conducted by two psychiatrists and diagnoses were based on concordance between independent assessments made by them. Diagnoses for all patients were made using DSM-III criteria. All subjects clinically required hospitalization for treatment of their affective disorder, and were inpatients for the period of the study. The subjects received a detailed physical examination and a battery of standard laboratory tests on their first day of hospitalization. Individuals were excluded from the study if there was evidence of any major physical illness, alcohol or other substance abuse, or contraindication to lumbar puncture. All subjects gave written informed consent to participate in the study after its nature and risks were explained to them. None of the patients received any psychotropic drugs for at least 2 weeks prior to the lumbar puncture. Through hospitalization, all patients received a low monoamine diet, and caffeine and alcohol were strictly proscribed.

\section{Methods}

Lumbar punctures took place between 9.00 and 10.00 a.m. following overnight fasting and at least $12 \mathrm{~h}$ bed rest. The lumbar punctures were performed with a fine needle in the left lateral decubitus position in the L4-L5 intervertebral space. About $12-13 \mathrm{ml}$ of CSF was collected in plypropylene tubes, immediately centrifuged and stored frozen in aliquots $<-20^{\circ} \mathrm{C}$ and kept at this temperature until analyzed.

HVA and 5-HIAA were assayed by the spectrofluorometric technique. While the currently more commonly employed gas chromatographic (GC)-mass spectrometric $(45,46)$ and high-performance liquid chromatographic (HPLC)-electrochemical detection (47) techniques are more sensitive and specific, HVA and 5-HIAA determinations using the spectrofluorometric method correlate very highly $(r=0.91)$ with those obtained by the former techniques, and the fluorometric method is considered adequate for most studies (48). HVA was assayed by Anden's method (49) and 5-HIAA by the method of Ashcroft \& Sharman (50). CSF sodium and potassium were measured by the flame-photometric method; calcium by Clark \& Collip's fluorometric method (51); and magnesium by Schacter's fluorometric method (52).

The Hamilton Depression Rating Scale $(53,54)$ and the Petterson Mania Rating Scale (55) were administered to all patients on the evening prior to the lumbar puncture; aggregate scores from these scales were employed as measures of depressive and manic symptomatology, respectively. The Petterson/Hamilton aggregate ratio was employed as a rough measure of the relative proportion of manic

Table 1

CSF biochemical profile of mania, mixed affective states, and major depression

\begin{tabular}{|c|c|c|c|c|c|c|}
\hline \multirow{2}{*}{ Biochemical parameter } & \multirow{2}{*}{ Manic } & \multirow{2}{*}{$\begin{array}{l}\text { Mixed affective } \\
\qquad n=14\end{array}$} & \multirow{2}{*}{$\begin{array}{c}\text { Depressed } \\
n=14\end{array}$} & \multicolumn{3}{|c|}{ Analysis of variance } \\
\hline & & & & $F$ & $\mathrm{df}$ & $P$ \\
\hline HVA (ng/ml) & $23.7 \pm 3.9^{*}$ & $20.2 \pm 10.1$ & $16.9 \pm 4.3$ & 3.42 & 2,39 & $<0.05$ \\
\hline 5-HLAA (ng/ml) & $42.8 \pm 8.4$ & $34.3 \pm 7.1$ & $31.5 \pm 10.5$ & 5.87 & 2,39 & $<0.05$ \\
\hline Sodium (mEq/l) & $145.9 \pm 8.4$ & $141.8 \pm 8.8$ & $137.1 \pm 9.7$ & 3.08 & 2,39 & 0.09 \\
\hline Potassium (mEq/l) & $2.89 \pm 0.49$ & $3.04 \pm 0.55$ & $2.86 \pm 0.54$ & 0.48 & 2.39 & NS \\
\hline Calcium $(\mathrm{mEq} / \mathrm{l})$ & $2.19 \pm 0.20$ & $2.26 \pm 0.29$ & $2.25 \pm 0.20$ & 0.34 & 2,39 & NS \\
\hline Magnesium (mEq/l) & $2.21 \pm 0.20$ & $2.24 \pm 0.14$ & $2.23 \pm 0.21$ & 0.16 & 2.39 & NS \\
\hline
\end{tabular}

*Values expressed as mean \pm standard deviation

Laboratory values for normal controls using same technique:

CSF HVA $\quad 25-35 \mathrm{ng} / \mathrm{ml}$

CSF 5-HIAA $\quad 50-60 \mathrm{ng} / \mathrm{ml}$

CSF sodium $\quad 120-145 \mathrm{mEq} / 1$

CSF potassium $2.5-4.8 \mathrm{mEq} / 1$

CSF calcium $\quad 2.0-2.5 \mathrm{mEq} / 1$

CSF magnesium $2.2-2.5 \mathrm{mEq} / \mathrm{l}$ 
to depressive symptoms and was used to clinically subcategorize the mixed affective group.

The three groups were initially compared on the six biochemical variables using analysis of variance (ANOVA). The manic and major depressive groups were next compared on these biochemical variables using post hoc $t$-tests, and based on differentiating biochemical variables, a set of discriminant functions was derived. This set of discriminant functions was applied to the manic and major depressive groups to evaluate its validity in the classification of affective patients. This same set of discriminant functions was then applied to the 14 subjects in the mixed affective group in an effort to dichotomize them. The subgroups derived from this analysis were next compared with each other, and with the manic and major depressive groups separately. The biochemical subgroups thus derived were compared with the previously obtained clinical subgroups to evaluate the overlap using the chi square test.

\section{Results}

There were no significant age, sex, height or weight differences between the three groups. No significant differences were found between the

Table 2

Discriminant functions on significant biochemical variables

\begin{tabular}{lcc}
\hline & Manic & Depressive \\
\hline Constant & -133.02 & -112.42 \\
HVA & 0.46622 & 0.14606 \\
5-HIAA & 0.46351 & 0.36406 \\
Sodium & 1.6112 & 1.5378 \\
General variance & 74408 & 198240 \\
$n$ & 14 & 14 \\
\hline
\end{tabular}

unipolar and bipolar depressed patients, and they could therefore be combined and analyzed together. Table 1 shows the results for the three groups for each of the CSF biochemical parameters. ANOVA revealed significant differences in HVA and 5-HIAA levels between the three groups, and a trend towards a significant sodium difference. The mean HVA, 5-HIAA, and sodium levels were all higher in the manic group than in the major depressive group; the mixed affective group occupied an intermediate position between the two groups on all these three biochemical variables. There were no major differences between the groups with respect to potassium, calcium, and magnesium levels.

The manic and major depressive groups were next compared on all the CSF biochemical variables using post hoc $t$-tests and were found to differ significantly with regard to HVA $(t=$ 4.30, $\mathrm{df}=26, P<0.001)$, 5-HIAA $(t=3.87$, $\mathrm{df}=26, P<0.001)$, and sodium $(t=2.46$, df $=26, P<0.05$ ) levels. Based on these differentiating biochemical variables, a set of discriminant functions was obtained (Table 2). This set of discriminant functions was applied to the 28 patients in the manic and major depressive groups and the subjects classified as manic or major depressive based on the discriminant function. This process resulted in the correct classification of 23 out of 28 patients $\left(\chi^{2}=11.71\right.$, df $=1, P<0.001)$ in these groups.

The same set of discriminant functions was then applied to the mixed affective group. Discriminant analysis of the mixed affective group identified two subgroups; one was classified as manic, and the other classified as major depressive. Both of these subgroups (the mixed manic and the mixed depressive) consisted of 7 patients each. These two subgroups were compared and

Table 3

Comparison of mixed affective subgroups

\begin{tabular}{lccccc}
\hline $\begin{array}{l}\text { SCF biochemical } \\
\text { parameters }\end{array}$ & Mixed manic & Mixed depressive & \multicolumn{3}{c}{ Two-tailed t-test } \\
& $n=7$ & $n=7$ & $t$ & $\mathrm{df}$ & $P$ \\
\hline HVA & $25.0 \pm 6.1$ & $15.5 \pm 4.3$ & 3.10 & 12 & $<0.01$ \\
5-HIAA & $39.2 \pm 6.6$ & $29.3 \pm 8.3$ & 2.30 & 12 & $<0.05$ \\
Sodium & $144.3 \pm 0.11$ & $139.4 \pm 6.3$ & 0.92 & 12 & NS \\
\hline
\end{tabular}


were found to differ significantly with regard to the CSF biochemical variables HVA and 5-HIAA (Table 3).

Table 4 shows the comparison of both these subgroups with the manic and major depressive groups. The mixed manic subgroup did not differ from the manic group with regard to any of the biochemical variables, but differed significantly from the major depressive group in terms of HVA, with a tendency towards significant difference with regard to 5-HIAA and sodium. Similarly, the mixed depressive subgroup did not differ from the major depressive group on any of the biochemical parameters; but it differed significantly from the manic group with regard to HVA and 5-HIAA, with a tendency towards a significant difference with regard to sodium.

The two mixed affective subgroups were next compared with regard to their clinical parameters (Table 5), using the Hamilton and Petterson

Table 4

Comparison of mixed affective subgroups with manic and depressive groups by two-tailed t-test

\begin{tabular}{|c|c|c|c|c|c|c|c|}
\hline & & \multicolumn{3}{|c|}{$\begin{array}{c}\text { Manic } \\
n=14\end{array}$} & \multicolumn{3}{|c|}{$\begin{array}{c}\text { Depressive } \\
n=14\end{array}$} \\
\hline & & $t$ & $\mathrm{df}$ & $P$ & $t$ & $\mathrm{df}$ & $P$ \\
\hline \multicolumn{8}{|c|}{ Mixed manic } \\
\hline \multirow[t]{3}{*}{$n=7$} & HVA & 0.56 & 19 & NS & 3.47 & 19 & $<0.01$ \\
\hline & 5-HLAA & -0.95 & 19 & NS & 1.71 & 19 & 0.10 \\
\hline & Sodium & -0.37 & 19 & NS & 1.47 & 19 & 0.15 \\
\hline \multicolumn{8}{|c|}{ Mixed depressive } \\
\hline \multirow[t]{3}{*}{$n=7$} & HVA & -4.3 & 19 & $<0.005$ & -0.69 & 19 & NS \\
\hline & 5-HIAA & -3.4 & 19 & $<0.01$ & -0.49 & 19 & NS \\
\hline & Sodium & -1.7 & 19 & 0.09 & 0.55 & 19 & NS \\
\hline
\end{tabular}

Thus the mixed manic subgroup was not different from the manic group, but differed from the depressive group with regard to HVA and showed a tendency towards significant differences in terms of 5-HIAA and sodium. Similarly, the mixed depressive subgroup did not differ from the depressive group, but differed from the manic group in terms of HVA and 5-HIAA, and showed a tendency rowards a significant difference with regards to sodium.

Table 5

Clinical parameters of mixed affective subgroups

\begin{tabular}{lccccc}
\hline & Mixed manic & Mixed depressive & \multicolumn{3}{c}{ Two-tailed t-test } \\
\cline { 5 - 6 } & $n=7$ & $n=7$ & $t$ & df & $P$ \\
\hline Mean Hamilton & 23.3 & 23.9 & 0.44 & 12 & NS \\
Mean Petterson & 26.0 & 21.2 & 3.91 & 12 & $<0.001$ \\
Mean Petterson/Hamilton ratio & 1.14 & 0.86 & 2.36 & 12 & $<0.05$ \\
\hline
\end{tabular}

Table 6

Relationship between clinical and biochemical subcategorization of mixed affective states

\begin{tabular}{|c|c|c|}
\hline & Biochemical mixed manic & Biochemical mixed depressive \\
\hline Clinical mixed manic & 7 & 2 \\
\hline Clinical mixed depressive & 0 & 5 \\
\hline chi square $=8.29$ & $P<0.005$ & \\
\hline
\end{tabular}

The Petterson/Hamilton ratio of 0.85 was used as the cut-off point to clinically subcategorize mixed affective states, with subjects having a Petterson/Hamilton ratio of $<0.85$ being classified as depressive, and those with a ratio $>0.85$ being clinically classified as manic. 
aggregate scores as measures of depressive and manic symptomatology, respectively. The Petterson/Hamilton ratio was employed as a crude measure of the ratio of manic to depressive symptomatology in each mixed affective patient. The two subgroups differed significantly with respect to the Petterson aggregate score and the Petterson/Hamilton ratio.

Based on the Petterson/Hamilton ratio, the mixed affective group was then subcategorized clinically. A Petterson/Hamilton aggregate ratio of 0.85 yielded the best clinical division between the two subgroups, with subjects having a ratio of $>0.85$ being classified as "clinically mixed manic", and patients with a ratio of $<0.85$ being classified as "clinically mixed depressive". Table 6 shows the relationship between the biochemical and clinical subcategorization of mixed affective states. All seven mixed affective subjects who were biochemically classified as manic were also clinically so classified. Of the mixed affective patients biochemically classified as major depressive, five were clinically so classified while two were classified as clinically manic. These data provided a clinical validation of the biochemical subcategorization of mixed affective states.

\section{Discussion}

Our finding of normal CSF HVA levels in mania is consistent with most previous studies $(39,40)$, as is the finding of decreased CSF HVA in major depression (26-33). Similarly, our finding of reduced 5-HIAA levels in both mania and major depression is also consistent with those of most studies $(21,30,31,41,42)$, with 5-HIAA levels significantly lower in major depression than in mania. While the CSF sodium levels were in the normal range in both mania and depression, as most commonly found (43), they were significantly lower in the depressive group than in the manic group. These results are consistent with the widely accepted view that disturbances in the CSF turnover of dopamine and serotonin, as reflected in the CSF levels of their metabolites HVA and 5-HIAA (respectively), may play a major role in the pathogenesis of affective disorders. The finding of lower CSF levels of sodium in depression than in mania is consistent with the hypothesis of a low rate of turnover of sodium by the nervous system in depressed patients (43), which was based on the finding of a low transfer rate of sodium from blood to CSF (56). The finding of normal CSF potassium, calcium, and magnesium levels, which were similar in both groups, is consistent with most previous reports (43). It should be noted that the 3 groups did not differ significantly with regard to such potentially confounding variables as age, sex, height, and weight.

The discriminant function derived from the differentiating biochemical variables correctly classified 23 out of 28 subjects in the manic and major depressive groups, suggesting that this technique is significantly accurate in the classification of affective patients as manic or depressive. This discriminant function subcategorized the mixed affective group into manic and depressive subgroups, each consisting of 7 patients, which differed significantly from one another with regard to HVA and 5-HIAA. Furthermore, the mixed manic subgroup did not differ from the original manic group, but differed significantly from the depressive group; similarly the mixed depressive subgroup resembled the depressive group, but differed significantly from the manic group. The two mixed affective subgroups clinically differed with regard to the ratio of manic to depressive symptomatology, and the clinical subcategorization of the mixed affective group coincided with the biochemical categorization in 12 out of 14 mixed affective patients.

These findings do not support the view of mixed affective states as a distinct homogeneous entity. Instead, they suggest that mixed affective states constitute a heterogeneous grouping, comprised of patients derived from the manic and major depressive categories (respectively). This conclusion is supported by a recent study of cerebral metabolic rates in mood disorders (57), which found a metabolic rate dichotomy between mania and depression, with the three mixed affective patients in the study showing a metabolic pattern similar to that of the depressed group.

The finding that mixed affective states do not exist as a separate entity, but are best viewed as 
either manic or depressed, removes one of the strongest criticisms against the bipolar model and eliminates the need for elaborate models to explain their existence. While Court $(11,15)$ postulated a "unipolar" model, with depression, mixed states and mania being on a continuum, and merely reflecting different severities of illness, Nunn (4) hypothesized that mixed affective states represented a superimposition on hypomania of a "second kind of depression", or the development of schizophrenia in the course of a manic-depressive illness. Himmelhoch $(14,18)$ suggested that mixed affective states occur either in severely ill, continuum-model, manic depressive patients, or in "true bipolar patients" whose illness was complicated by a second neuropsychiatric condition such as sedative abuse or epileptic changes. Thus, the observation that mixed affective states do not exist separately, strongly suggests that the unipolar model does not represent a viable conceptualization of the nature of manic-depressive illness, since this model predicts and is based on the existence of such states. This finding also eliminates the need to devise other elaborate models to explain mixed affective states. While it will continue to be both interesting and important to investigate why some patients exhibit mixed affective phenomenology and others do not, these data suggest that it seems unlikely that the answer resides in the existence of mixed affective states as a separate entity, distinct from mania and major depression.

The clinical implication of these findings is that mixed affective states are best treated as either manic or depressed. Although their clinical subcategorization is difficult, our Petterson/ Hamilton aggregate ratio findings suggest that this subcategorization really exists. Clearly, better instruments to make this clinical distinction need to be devised. It has been suggested that mixed affective states respond poorly to treatment and represent a treatment-resistant condition $(1,12,18,58)$. Perhaps this may be attributable to inappropriate treatment of this condition because of a failure to separate this group into its predominant manic or depressive subgroup and treat accordingly. This hypothesis merits testing.
Several methodological issues deserve comment. Our inability to assay MHPG is a majorshortcoming of this study. While the spectrofluorometric technique for the assay of HVA and 5-HIAA is less sensitive than newer methods, it correlates well with them and is considered adequate for most studies (48). The unipolar and bipolar depressed subjects were analyzed together, as no significant differences were found between them. In particular, it has been reported that there is no significant difference between them with regard to the three CSF monoamine metabolites (33). The probenecid technique was not employed, as it has been suggested that instead of removing a source of variance in CSF metabolite studies, this technology introduces new and intriguing complexities (59). Finally, replication of the discriminant function would be desirable.

Although the findings of this study clearly need to be replicated and extended, measuring other biochemical variables (especially MHPG), and employing more sophisticated methods of assay, the results of this study indicate that mixed affective states do not exist as a separate entity and are actually composed of two subgroups derived from the major depressive and manic categories, respectively.

\section{Acknowledgement}

We thank Cynthia Pomerleau, Ph. D., for editorial assisance.

\section{References}

1. Kraepelin E. Lectures on clinical psychiatry: Lecture VIII (a facsimile of the 1904 edition). New York: Hafner Publishing, 1968: 68-76.

2. Kraepelin E. Manic-depressive insanity and paranoia. Edinburgh: E \& S Livingston, 1921.

3. Redlich F C, Freedman DX. The theory and practice of psychiatry. New York: Basic Books, 1966: 533-563.

4. Nunn $\mathrm{C} M \mathrm{M}$. Mixed affective states and the natural history of manic-depressive psychosis. Br J Psychiatry 1979: 134:153-160.

5. Gardner R. Mechanisms in manic-depressive disorder: an evolutionary model. Arch Gen Psychiatry 1982:39:14361441.

6. Schildkraut J J. The catecholamine hypothesis of affective disorders: a review of supporting evidence. Am J Psychiatry 1965:122:509-522.

7. American Psychiatric Association. Diagnostic and statisti- 
cal manual of mental disorders (DSM-III). 3rd ed. Washington, DC: American Psychiatric Association, 1980.

8. World Health Organization. Mental disorders: glossary and guide to their classification in accordance with the ninth revision of the International Classification of Diseases. Geneva: WHO, 1978.

9. Kotin J, Goodwin F K. Depression during mania: clinical observations and theoretical implications. Am J Psychiatry 1972:129:55-62.

10. Bunney W F, Murphy D L, Goodwin F K. The switch process in manic-depressive illness. Parts 1-3. Arch Gen Psychiatry 1972:27:295-317.

11. Court J H. Manic depressive psychosis: an alternative conceptual model. Br J Psychiatry 1968:114:1523-1530.

12. Himmelhoch $\mathbf{J} H$, Mulla $D, N e i l J F$, et al. Incidence and significance of mixed affective states in a bipolar population. Arch Gen Psychiatry 1976:33:1062-1066.

13. Himmelhoch J H, Coble P, Kupfer D J, et al. Agitated psychotic depression associated with severe hypomanic episodes. Am J Psychiatry 1976:133:765-771.

14. Himmelhoch $\mathbf{J}$ H. Mixed states, manic-depressive illness, and the nature of mood. Psychiatr Clin North Am 1979:2:449-459.

15. Court $\mathrm{J} \mathrm{H}$. The continuum model as a resolution of paradoxes in manic-depressive psychosis. $\mathrm{Br} \mathrm{J}$ Psychiatry 1972:120:133-141.

16. Benoit $G$. The origin, fate and current interest of mixed states. Confront Psychiatry 1976:14:105-110.

17. Sitaram N, Gillin J C, Bunney W E. The switch process in manic-depressive illness: circadian variation in time of switch and sleep and manic ratings before and after switch. Acta Psychiatr Scand 1978:58:267-278.

18. Himmelhoch $J \mathrm{H}$, Garfinkel M E. Sources of lithium resistance in mixed mania. Psychopharmacol Bull 1986: 22:613-620.

19. Post R M, Stoddard F J, Gillin C J, et al. Alterations in motor activity, sleep, and biochernistry in a rapidly cycling manic-depressive patient. Arch Gen Psychiatry 1977:34: 470-477.

20. Bunney $\mathrm{J}_{\mathrm{r}} \mathrm{W} E$, Davis J M. Norepinephrine in depressive reactions. Arch Gen Psychiatry 1965:13:483-494.

21. Ȧsberg $M$, Thorén $P$, Träskman $L$, et al. Serotonin depression - a biochemical subgroup within the affective disorders? Science 1976:191:478-480.

22. Maas J W. Biogenic amines and depression. Arch Gen Psychiatry 1975:32:1357-1364.

23. Van Praag H M. Significance of biochemical parameters in the diagnosis, treatment, and prevention of depressive disorders. Biol Psychiatry 1977:12:101-131.

24. Post RM, Ballenger J C, Goodwin F K. Cerebrospinal fluid studies of neurotransmitter function in manic and depressive illness. In Wood J H, ed. Neurobiology of cerebrospinal fluid. Vol. 1. New York, Plenum Press, 1980.

25. Shopsin B. Manic illness. New York: Raven Press, 1979:137-162.

26. Bowers M B, Heninger G R, Gerbode F. Cerebrospinal fluid 5-hydroxyindoleacetic acid and homovanillic acid in psychiatric patients. Int J Psychopharmacology 1969: 8:255-262.

27. Roos B E, Sjöström R. 5-hydroxyindoleacetic acid and homovanillic acid levels in the cerebrospinal fluid after probenecid application in patients with manic depressive psychosis. Pharmacol Clin 1969:1:153-155.

28. Papeschi R, McClure D J. Homovanillic acid and 5-hydroxyindoleacetic acid in cerebrospinal fluid of depressed patients. Arch Gen Psychiatry 1971:25:354-358.

29. Goodwin F K, Post R M, Dunner D L, et al. Cerebrospinal fluid amine metabolites in affective illness: the probenecid technique. Am J Psychiatry 1973:130:73-79.

30. Mendels J, Frazer A, Fitzgerald R G, et al. Biogenic amine metabolites in cerebrospinal fluid of depressed and manic patients. Science 1972:175:1380-1382.

31. Åsberg M, Bertilsson L, Mảrtensson $B$, et al. CSF monoamine metabolites in melancholia. Acta Psychiatr Scand 1984:69:201-219.

32. Roy A, Pickar D, Linnoila M, et al. Cerebrospinal fluid monoamine and monoamine metabolite concentrations in melancholia. Psychiatr Res 1985:15:281-292.

33. Koslow S H, Maas J W, Bowden C L, et al. CSF and urinary biogenic amines and metabolites in depression and mania. A controlled univariate analysis. Arch Gen Psychiatry 1983:40:999-1010.

34. Banki C M, Arato M. Relationship between cerebrospinal fluid amine metabolites, neuroendocrine findings and personality dimensions in psychiatric patients. Acta Psychiatr Scand 1983:67:272-280.

35. Gerner R H, Fairbanks L, Anderson G M, et al. CSF neurochemistry in depressed, manic and schizophrenic patients compared with that of normal controls. Am J Psychiatry 1984:141:1533-1540.

36. Gjerris A, Werdelin L, Gjerris F, et al. CSF-amine metabolites in depression, dementia and in controls. Acta Psychiatr Scand 1987:75:619-628.

37. Sjöström R, Roos B E. 5-hydroxyindoleacetic acid and homovanillic acid in cerebrospinal fluid in manic depressive psychosis. Eur J Clin Pharmacol 1972:4:170-176.

38. Berger P A, Faull K F, Kilkowski J, et al. CSF monoamine metabolites in depression and schizophrenia. Am J Psychiatry 1980:137:174-180.

39. Post R M, Jimerson D C, Bunney $J_{\Gamma} W E$, et al. Dopamine and mania: behavioral and biochemical effects of the dopamine receptor blocker pimozide. Psychopharmacology 1980:67:297-305.

40. Swann A C, Secunda S, Davis J M, et al. CSF monoamine metabolites in mania. Am J Psychiatry 1983:140:296-400.

41. Banki C M. Correlation between cerebrospinal fluid amine metabolites and psychomotor activity in affective disorders. J Neurochem 1977:28:255-257.

42. Ashcroft G W, Crawford T T B, Eccleston D. 5-hydroxyindole compounds in the cerebrospinal fluid of patients with psychiatric or neurological diseases. Lancet 1966: ii: 1049-1050.

43. Mellerup E T, Rafaelsen O J. Electrolyte metabolism and manic-melancholic disorder. In: Van Praag H M, Lader M H, Rafaelsen O J, Sachar E J, eds. New York: Marcel \& Dekker, 1981.

44. Ueno $Y$, Aoki N, Yabuki $T$, et al. Electrolyte metabolism in blood and cerebrospinal fluid in psychoses. Folia Psychiatr Neurol Jpn 1961:15:304-326.

45. Fri C G, Wiesel F A, Sedvall G. Simultaneous quantifica- 
tion of homovanillic acid and 5-hydroxyindoleacetic acid in cerebrospinal fluid by mass fragmentography. Life Sci 1974:14:2469-2480.

46. Swahn C G, Sandgarde B, Wiesel F A, et al. Simultaneous determination of the three major monoamine metabolites in brain tissue and body fluids by a mass spectrometric method. Psychopharmacology 1976:48:147-152.

47. Scheinin $M$, Chang $W$, Kirk $K$, et al. Simultaneous determination of 3-methoxy-4-hydroxy phenylglycol, 5-hydroxyindoleacetic acid, and homovanillic acid in cerebrospinal fluid with high performance liquid chromatography using electrochemical detection. Anal Biochem 1983:131:246-253.

48. Jimerson D C, Gordon E K, Post $R \mathrm{M}$, et al. HVA in human CSF: comparison of fluorometry and gas chromatography-mass spectrometry. Commun Psychopharmacol 1978:2:343-350.

49. Anden N, Roos B E, Werdinus B. On the occurrence of HVA in brain and cerebrospinal fluid and determination by fluorometry. Life Sci 1963:2:448-458.

50. Ashcroft GW, Sharman D F. Drug induced changes in the concentration of 5OH-Indolyl compounds in the CSF and caudate nucleus. Br J Pharmacol 1962:19:153-160.

51. Clark E P, Collip JB. A study of the Tisdall method for the determination of blood serum calcium with a suggested modification. J Biol Chem 1925:63:462-464.

52. Schacter D. The fluorometric estimation of magnesium in serum and in urine. J Lab Clin Med 1959:54:763-768.
53. Hamilton M. A rating scale for depression. J Neurol Neurosurg Psychiatr 1960:23:56-62.

54. Hamilton M. Development of a rating scale for primary depressive illness. Br J Soc Clin Psychol 1976:6:278-296.

55. Petterson U, Fyro B, Sedvall G. A new rating scale for the longitudinal rating of manic states. Acta Psychiatr Scand 1973:49:248-256.

56. Coppen A. Mineral metabolism in affective disorders. Br J Psychiatry 1965:111:1133-1142.

57. Baxter Jr L R, Phelps M E, Mazziota J C, et al. Cerebral metabolic rates for glucose in mood disorders. Arch Gen Psychiatry 1985:42:441-447.

58. Dunner D L. Clinical characteristics of lithium prophylaxis failure. Arch Gen Psychiatry 1974:30:229-233.

59. Cowdry R W, Ebert M H, van Kammen D P, et al. Cerebrospinal fluid probenecid studies: a reinterpretation. Biol Psychiatry 1983:18:1287-1299.

Address

Rajiv Tandon, M.D.

Department of Psychiatry

UH-9C-9050

University of Michigan Medical Center

1500 E. Medical Center Drive

Ann Arbor, MI 48109-0120

USA 